\title{
Two-dimensional Dispersive Beam Steerer Fabricated on Silicon-On-Insulator.
}

\author{
Karel Van Acoleyen, Wim Bogaerts and Roel Baets \\ Department of Information Technology (INTEC) \\ Photonics Research Group \\ Ghent University - imec, Belgium \\ Email: karel.vanacoleyen@intec.ugent.be
}

\author{
Hendrik Rogier \\ Department of Information Technology (INTEC) \\ Electromagnetics Group \\ Ghent University, Belgium
}

\begin{abstract}
A two-dimensional beam steerer on silicon-oninsulator is presented. Steering ranges of $5.5^{\circ}$ in one direction and $50^{\circ}$ in the other direction have been shown for a wavelength shift of 40nm. The largest measured sensitivity was $10.7^{\circ}$ per nanometer wavelength shift.
\end{abstract}

\section{INTRODUCTION}

One of the key elements in free-space optical applications is beam steering [1]. Beam steering can be achieved with several methods such as MEMS (Micro ElectroMechanical Systems), movable mirrors or optical phased arrays (OPAs). The latter have the advantage that they are robust and insensitive to acceleration. When using an OPA, there is a need to tune the phase of each pixel. A popular way of performing this is, for example, using liquid crystals. In [2], an integrated OPA on silicon-on-insulator (SOI) has been demonstrated using thermo-optic phase tuning. Beam steering can, however, also be performed by wavelength tuning. When using diffractive elements, the direction of emission can be controlled by wavelength tuning. A two-dimensional space can then be scanned by using two dispersive elements. This principle has been shown in [3].

In this paper, we present a fully integrated approach to perform two-dimensional wavelength scanning. Therefore we make use of the silicon photonics platform using SOI. The component presented here scans a two-dimensional space using wavelength tuning. The principle is that the beam direction is scanned quickly in one direction while it changes slowly in the other direction when changing the wavelength. The component is quite similar to an Arrayed Waveguide Grating (AWG) in which the last star coupler section has been replaced by gratings for outcoupling. The grating and the AWG delay lines are the two dispersive elements to perform beam steering. Such dispersive components could find applications in, for example, spectroscopy applications or in optical wireless sensor networks. In the latter, the component would act as a cheap node, while the base station would perform wavelength steering and tuning to address the right node. This would result in a complex base station design but cheap optical nodes that can be addressed. Another application could be free space WDM (Wavelength Division Multiplexing) networks, where a room is divided into different sections that are addressed by a different wavelength.
Next, we discuss the design and fabrication of the component. In Section 3 the measurement results are given. Section 4 gives the conclusion.

\section{DESIGN AND FABRICATION}

The structure (shown schematically in Fig. 1) was fabricated on SOI at imec, Leuven using standard CMOS (Complementary Metal Oxide Semiconductor) processes [4], [5]. The buried oxide layer layer is $2 \mu \mathrm{m}$ and the silicon top layer is $220 \mathrm{~nm}$ high. A deep etch of $220 \mathrm{~nm}$ was used to etch the waveguides and the star coupler and a shallow etch of $70 \mathrm{~nm}$ to etch the grating couplers and tapering sections of the star coupler. Light is guided from an optical fiber into the structure using a grating coupler for near vertical coupling of the TElike mode [6]. The waveguide is then adiabatically tapered to a $450 \mathrm{~nm}$ wide photonic wire which is the input waveguide shown in Fig. 1. Light is then split through a star coupler into 16 waveguides. There is a fixed delay length $\Delta L$ between each waveguide. At the end of each waveguide, the photonic wire tapers to a $800 \mathrm{~nm}$ wide wire on which a grating is etched.

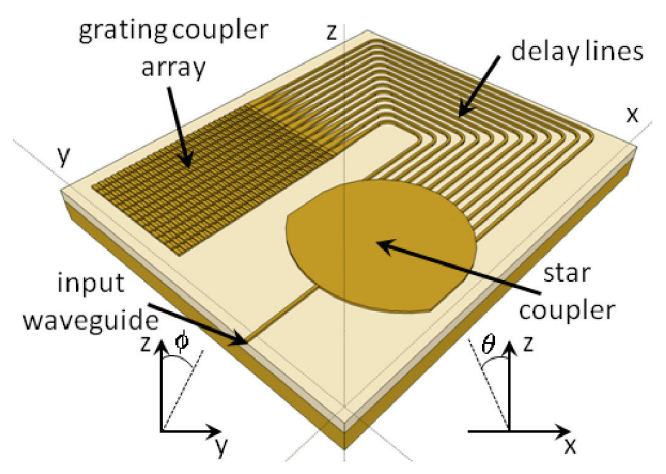

Fig. 1. Two-dimensional beam steerer fabricated on SOI.

The grating is the first dispersive element that will determine the steering in the $\theta$-direction (Fig. 1), given by the grating equation:

$$
\sin \theta=\frac{\Lambda_{g r} n_{e f f, g r}-\lambda_{0}}{n_{c t} \Lambda_{g r}}
$$

with $\Lambda_{g r}$ the period of the grating, $\lambda$ the free-space wavelength, $n_{e f f, g r}$ the effective index of the guided mode in the 
grating area and $n_{c t}$ the refractive index of the background which is air $\left(n_{c t}=1\right)$ in this case. The steering speed is given by

$$
\frac{\mathrm{d} \theta}{\mathrm{d} \lambda} \approx \frac{\mathrm{d} \sin \theta}{\mathrm{d} \lambda}=\frac{\mathrm{d} n_{e f f, g r}}{\mathrm{~d} \lambda}-\frac{1}{\Lambda_{g r}}
$$

where the approximation is valid when the angle $\theta$ is relatively small. Note the dispersion factor $\mathrm{d} n_{\text {eff,gr }} / \mathrm{d} \lambda$, which cannot be neglected. The dispersion is about $-8.9 \times 10^{-4} / \mathrm{nm}$ for a $70 \mathrm{~nm}$ deep etched grating in a $800 \mathrm{~nm}$ wide waveguide.

The delay lines will now determine the steering in the $\phi$ direction (Fig. 1). The grating couplers are placed in an $N$ array configuration with positions $\mathbf{s}_{n}=n \Lambda_{y} \mathbf{u}_{y}$ with $\Lambda_{y}$ the spacing of the elements in the y-direction and $n=0 \ldots N-1$. The far field can be calculated by multiplication of the far field of one grating coupler with the array factor $T$ [7]:

$$
T=\sum_{n=0}^{N-1} A_{n} e^{-j \beta_{n}} e^{j \mathbf{k} \cdot \mathbf{s}_{n}}=\sum_{n=0}^{N-1} A_{n} e^{-j \beta_{n}} e^{j k_{0}\left[n \Lambda_{y} \sin \phi\right]}
$$

where $\beta_{n}$ is the phase delay between the elements, $\mathbf{k}$ is the wave vector (in air) with magnitude $k_{0}$ and $A_{n}$ is the amplitude of each element which is further assumed to be 1 . The length difference $\Delta L$ results in a phase delay of:

$$
\beta_{n}=n_{e f f} \frac{2 \pi}{\lambda} n \Delta L
$$

with $n_{\text {eff }}$ the effective index of the fundamental TE-like mode. The array factor can then be calculated in closed form:

$T=e^{j\left[\left(k_{0} \Lambda_{y} \sin \phi-k_{0} n_{e f f} \Delta L\right) \frac{N-1}{2}\right]} \frac{\sin \left(N \frac{k_{0} \Lambda_{y} \sin \phi-k_{0} n_{\text {eff }} \Delta L}{2}\right)}{\sin \left(\frac{k_{0} \Lambda_{y} \sin \phi-k_{0} n_{e f f} \Delta L}{2}\right)}$

The array factor is maximum in the $\phi$-direction for

$$
\sin \phi=q \frac{\lambda}{\Lambda_{y}}+\frac{n_{e f f} \Delta L}{\Lambda_{y}}
$$

with $\mathrm{q}$ an integer. Due to the delay lines, the absolute value of $\mathrm{q}$ will be large. The beams will shift at a rate of

$$
\frac{\mathrm{d} \phi}{\mathrm{d} \lambda} \approx \frac{\mathrm{d} \sin \phi}{\mathrm{d} \lambda}=\frac{q}{\Lambda_{y}}+\frac{\mathrm{d} n_{\text {eff }}}{\mathrm{d} \lambda} \frac{\Delta L}{\Lambda_{y}}
$$

where the angle $\phi$ is assumed to be relatively small. Note that $q$ will be negative so that the beam will shift in the negative $\phi$-direction for increasing wavelength. To reduce the effect of phase noise in the delay lines due to fabrication tolerances, the delay lines are tapered to a $800 \mathrm{~nm}$ wide waveguides. The effective index at $\lambda=1550 \mathrm{~nm}$ for this wire is $n_{\text {eff }} \approx 2.648$. There are two main contributions to the dispersion: material dispersion and waveguide dispersion. Whereas the former is relatively weak for the wavelength range considered here, the latter can have a significant influence due to the extremely high confinement in the small photonic wires. The change of $n_{\text {eff }}$ with wavelength is negative as well and is about $-8.2 \times 10^{-4} / \mathrm{nm}$ around $\lambda=1550 \mathrm{~nm}$ for a $800 \mathrm{~nm}$ wide wire. This influence is reduced by having a wider waveguide. For comparison, this dispersion value is about $-0.013 / \mathrm{nm}$ in a $450 \mathrm{~nm}$ wide wire. However, the factor $\Delta L / \Lambda_{y}$ can become large so that the influence of dispersion cannot be neglected. Different wavelength scanners were fabricated and measured. The gratings were etched in a $800 \mathrm{~nm}$ wide wire with a period of $670 \mathrm{~nm}$ and a fill factor of $50 \%$. The effective index of the grating area is then about 2.48. Eq. (1) then gives a mean outcoupling angle $\theta_{0}=9.6^{\circ}$ at $\lambda=1550 \mathrm{~nm}$. The steering speed given by Eq. (2) is then $\mathrm{d} \theta / \mathrm{d} \lambda \approx 0.137^{\circ} / \mathrm{nm}$. The spacing of the gratings was $\Lambda_{y}=2 \mu \mathrm{m}$. The steering speed in the $\phi$-direction given by Eq. (7) depends mainly on the length difference $\Delta L$ and is summarized in Table I for the fabricated components.

\begin{tabular}{cccc}
$\Delta L(\mu \mathrm{m})$ & $\mathrm{q}$ & $\mathrm{d} \phi / \mathrm{d} \lambda\left({ }^{\circ} / \mathrm{nm}\right)$ (theory) & $\mathrm{d} \phi / \mathrm{d} \lambda\left({ }^{\circ} / \mathrm{nm}\right)$ (meas.) \\
\hline 29.2 & -50 & -2.12 & -2.16 \\
58.5 & -100 & -4.24 & -4.37 \\
87.7 & -150 & -6.36 & -6.47 \\
117.0 & -200 & -8.47 & -8.69 \\
146.2 & -250 & -10.60 & -10.72 \\
\hline
\end{tabular}

TABLE I

THEORETICAL AND MEASURED RESULTS OF THE FABRICATED COMPONENTS.

\section{Measurement Results}

A Fourier imaging setup was used to investigate the far field pattern of the components as shown in Fig. 2 [8]. The far field is imaged onto the back-focal plane of a microscope objective (MO). In this plane, one point corresponds to a specific direction of emission. This plane is then brought back to the infrared camera by means of two lenses. The numerical aperture (NA) of the microscope objective determines the maximum direction of emission that is captured by the measurement setup. An MO with a NA of 0.5 was used meaning that the maximum angle with respect to the normal of the surface that can be measured is $30^{\circ}$.

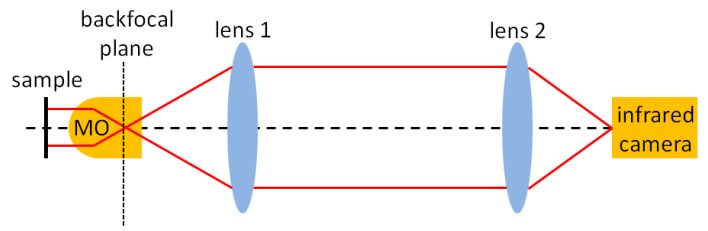

Fig. 2. A Fourier imaging setup was used to measure the far field of the beam steerers.

Fig. 3 shows the far field pattern of one of the beam steerers with $q=-250$ at ten different wavelengths. The FWHMwidth (full-width-half-maximum) is about $2.5^{\circ}$ in both the $\theta$ and $\phi$-direction. Due to the spacing of $2 \mu \mathrm{m}$, there is more than one lobe emitted by the array, not visible in Fig. 3. These are spaced about $\operatorname{asin}\left(\lambda / \Lambda_{\mathrm{y}}\right)$ which is about $50.8^{\circ}$ at a wavelength of $1550 \mathrm{~nm}$.

In order to scan the complete $2 \mathrm{D}$ space, the beam should shift $50^{\circ}$ in the $\phi$-direction when it has shifted $2.5^{\circ}$ in the $\theta$-direction (as the FWHM beam width is $2.5^{\circ}$ ). The latter happens over a wavelength range of about $18 \mathrm{~nm}$. The 


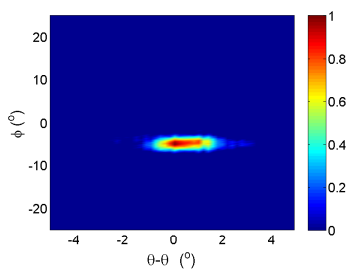

(a) $\lambda=1545 \mathrm{~nm}$

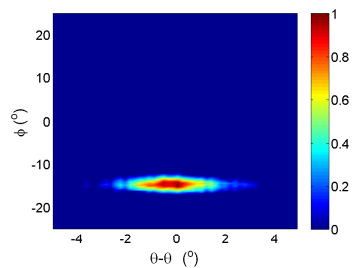

(f) $\lambda=1550 \mathrm{~nm}$

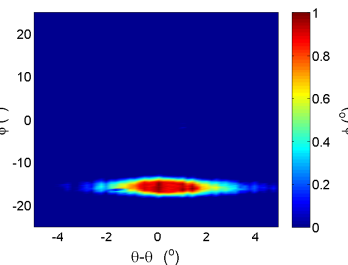

(b) $\lambda=1546 \mathrm{~nm}$

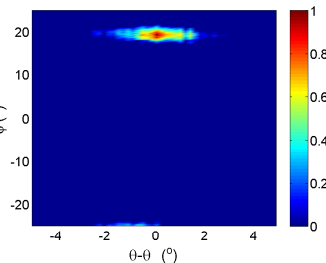

(g) $\lambda=1551 \mathrm{~nm}$

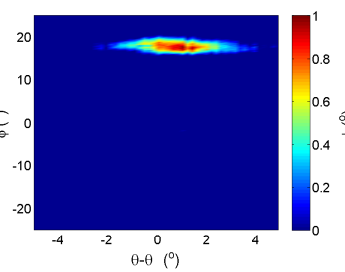

(c) $\lambda=1547 \mathrm{~nm}$

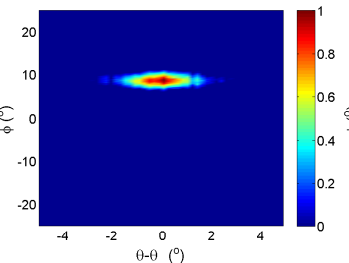

(h) $\lambda=1552 \mathrm{~nm}$

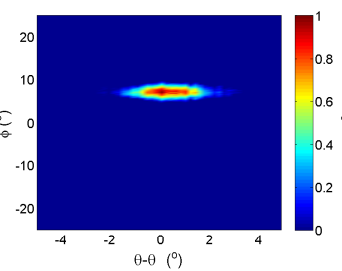

(d) $\lambda=1548 \mathrm{~nm}$

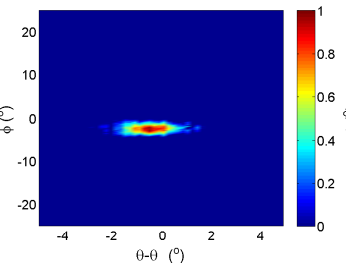

(i) $\lambda=1553 \mathrm{~nm}$

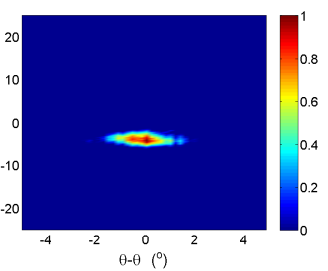

(e) $\lambda=1549 \mathrm{~nm}$

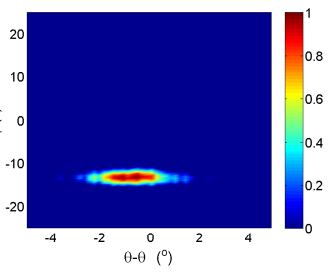

(j) $\lambda=1554 \mathrm{~nm}$

Fig. 3. Far field of the 2D beam steerer of order $q=-250$ at 10 different wavelengths.

minimum steering speed in the $\phi$-direction should thus be $2.78^{\circ} / \mathrm{nm}$. The results of the steering speed in the $\phi$-direction is given in Table I. A good agreement with the theoretical expected values can be seen.

The steering speed in the $\theta$-direction is the same for all components and was measured to be $0.137^{\circ} / \mathrm{nm}$ which corresponds to the theoretical value. The position of the beam for two of the fabricated components can be found in Fig. 4. The $\theta$ angle varies slowly while the $\phi$ angle changes quickly when changing the wavelength. At each jump, we focus on a different lobe emitted by the grating array. This lobe then shifts out of the measurement range until a new lobe appears.

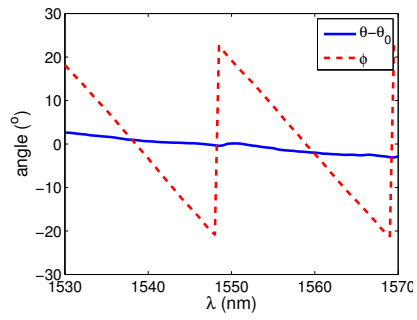

(a) $q=-50$

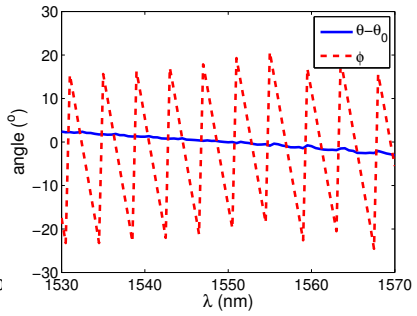

(b) $q=-250$
Fig. 4. Position of the beam for different orders $q$ of the delay length $\Delta L$.

The beam width can be decreased in the $\phi$-direction by having more waveguides. The beam width in the $\theta$-direction depends on the strength of the grating. This strength is strongly dependent on the grating etch. Having an etch of less than $70 \mathrm{~nm}$ would result in a weaker grating with a longer outcoupling length and thus a narrower beam. This would increase the sensitivity of the component.

\section{CONCLUSION}

A two-dimensional beam steerer on SOI has been presented. Steering ranges of about $5.5^{\circ}$ in the $\theta$-direction and $50^{\circ}$ in the $\phi$-direction were measured for a wavelength shift of $40 \mathrm{~nm}$. The maximum measured sensitivity of shift in the $\phi$-direction was $10.7^{\circ} / \mathrm{nm}$. This sensitivity can be further increased by increasing the delay lengths resulting in a faster steering speed or by having more waveguides resulting in a narrower beam.

\section{ACKNOWLEDGMENT}

Karel Van Acoleyen and Wim Bogaerts acknowledge the Research Foundation - Flanders (FWO) for a research grant and a postdoctoral fellowship, respectively.

\section{REFERENCES}

[1] P. F. McManamon, P. J. Bos, M. J. Escuti, J. Heikenfeld, S. Serati, H. K. Xie, and E. A. Watson, "A Review of Phased Array Steering for NarrowBand Electrooptical Systems," Proceedings of the IEEE, vol. 97, no. 6, pp. 1078-1096, 2009.

[2] K. Van Acoleyen, W. Bogaerts, J. Jágerská, N. Le Thomas, R. Houdré, and R. Baets, "Off-chip beam steering with a one-dimensional optical phased array on silicon-on-insulator," Optics Letters, vol. 34, no. 9, pp. 1477-1479, 2009.

[3] T. Chan, E. Myslivets, and J. E. Ford, "2-Dimensional beamsteering using dispersive deflectors and wavelength tuning," Optics Express, vol. 16, no. 19 , pp. $14617-14628,2008$.

[4] ePIXfab, "The silicon photonics platform," http://www.epixfab.eu/, 2009.

[5] S. K. Selvaraja, P. Jaenen, W. Bogaerts, D. Van Thourhout, P. Dumon, and R. Baets, "Fabrication of Photonic Wire and Crystal Circuits in Siliconon-Insulator Using 193-nm Optical Lithography," Journal of Lightwave Technology, vol. 27, no. 18, pp. 4076-4083, 2009.

[6] G. Roelkens, D. Vermeulen, D. Van Thourhout, R. Baets, S. Brision, P. Lyan, P. Gautier, and J. M. Fedeli, "High efficiency diffractive grating couplers for interfacing a single mode optical fiber with a nanophotonic silicon-on-insulator waveguide circuit," Applied Physics Letters, vol. 92, no. 13, p. 131101, 2008.

[7] C. A. Balanis, "Antenna Theory, Analysis and Design," John Wiley and Sons, Inc, 1982.

[8] N. Le Thomas, R. Houdré, M. V. Kotlyar, D. O'Brien, and T. E. Krauss, "Exploring light propagating in photonic crystals with Fourier optics," Journal of the Optical Society of America B, vol. 24, no. 12, pp. 29642971, 2007. 